\title{
Multimodal Methods for In Situ Transmission Electron Microscope
}

\author{
Renu Sharma, Wei-Chang Yang and Canhui Wang \\ National Institute of Standards and Technology, Gaithersburg, Maryland, United States
}

The application of transmission electron microscopy (TEM) related techniques have extended from ex situ nanoscale characterization of structure and chemistry of reaction products to dynamic measurements of nanostructures during reaction processes. Commercially-available modified TEM specimen holders and TEM columns (environmental scanning-transmission electron microscope or ESTEM) are being routinely employed to follow the structural and chemical changes at elevated temperatures and under controlled atmospheres. The combination of atomic-resolution images and high spatial and energy resolution has successfully revealed the functioning of catalyst nanoparticles. We have been involved in advancing technical and analytical techniques for unveiling gas-solid interactions at nanoscale.

We have incorporated a free-standing, light focusing system in the ESTEM to excite and collect vibrational and optical spectroscopies under reactive environments. This bimodal data collection enables the acquisition of vibrational spectroscopy data concurrently with electron microscopy data, (imaging, diffraction, electron energy-loss spectroscopy(EELS)), under identical environment, and expands the spatial resolution from nanoscale to microscale.[1] For example, we have collected Raman signals from $\approx 80 \mu \mathrm{m}^{2}$ areas, while collecting atomic-resolution videos from $200 \mathrm{~nm}^{2}$ area, during in situ growth of single-walled carbon nanotubes (SWCNT) at $\approx 625{ }^{\circ} \mathrm{C}$ in $\approx 0.005 \mathrm{~Pa}_{\text {of }} \mathrm{C}_{2} \mathrm{H}_{2}$.[2]

Recently, we have applied localized surface plasmon (LSP) resonance energy to initiate a chemical reaction at room temperature.[3] The energy barrier for most of the chemical reactions is overcome by heating the reactants in the presence of a suitable catalyst, where the thermal energy provided by heat helps in overcoming the reaction barrier that has been reduced by the adsorption of the reactant molecules on the catalyst nanoparticle surfaces. In recent years, LSP resonance energy has been used to replace the thermal energy to initiate reactions at low temperatures, even at room temperature, mimicking photocatalysis.[4,5] We leverage on the ability of high energy electrons to excite all of the LSP modes simultaneously, and provide high spatial resolution in the nanometer range to resolve the energy loss probability, measured using EELS that indicates the location-specific coupling efficiency of the electron excitation of the LSP modes at specific resonance energies on the plasmonic nanoparticle.[6] Moreover, core-loss EELS can be used to identify gas adsorption sites and solid reaction products.

We use a monochromated electron source in an environmental scanning-transmission electron microscope (ESTEM), combined with metallic nanoparticle boundary element method (MNPBEM)[7] to characterize various LSP modes and their coupling efficiency distribution within the shape and sizecontrolled $\mathrm{Au}$ and $\mathrm{Al}$ nanoparticles. In situ core-loss EELS was used to observe $\mathrm{CO}$ adsorption on the $\mathrm{Au}$ surfaces using carbon k-edge after removing the spectral contribution of environmental $\mathrm{CO}$ from individual pixels in a spectrum image. Low-loss EELS map from $1.8 \mathrm{eV}$ to $3.8 \mathrm{eV}$ showed that the electron beam position for exciting the dipole mode most ecciciently is located at the nanoprsim corner and has an energy shift in $\mathrm{CO}$ environment $\left(\mathrm{P}_{\mathrm{CO}} \approx 110 \mathrm{~Pa}\right)$ that disappears after $\mathrm{CO}$ evacuation, indicating a change in electron density of the Au particle as $\mathrm{CO}$ is adsorbed and desorbed at the selective edges, respectively. Carbon deposits, detected by core-loss EELS at the cantilevered corner edge after evacuating CO, were produced from room-temperature $\mathrm{CO}$ disproportionation $\left(2 \mathrm{CO}_{(g)} \rightarrow \mathrm{CO}_{2(g)}+\mathrm{C}_{(s)}\right)$, mediated by the LSP resonance excited by high energy electrons on $\mathrm{Au}$ nanoprism, Therefore, the active sites on $\mathrm{Au}$ 
nanoprisms, identified using EELS, are located where the preferable $\mathrm{CO}$ adsorption sites and the locations of maximum field amplitude superimpose.

We then apply this method to initiate the reverse reaction at room temperature. Since the reported energy range (between $2.91 \mathrm{eV}$ and $3.9 \mathrm{eV}$ ) required for the reverse reaction to proceed is higher than the resonance energy available in Au nanoparticles, we selected Al instead. Multimode LSP resonance energies of $\mathrm{Al}$ nanoparticles, spanning from $2 \mathrm{eV}$ and $8 \mathrm{eV}$, are simultaneously excited by high energy electrons. Suspensions of Al nanoparticles and graphite flakes, loaded on lacey carbon TEM grids, were introduced in the ESTEM column. Graphite flakes in contact with $\mathrm{Al}$ nanoparticles etched away in $\mathrm{CO}_{2}$ environment under an electron flux of $\approx 9.1 \times 10^{-6} \mathrm{nA} \cdot \mathrm{nm}^{-2}$. Thickness maps $(t / \lambda)$ were used to obtain the number of carbon atoms etched as a function of (a) distance away from the nanoparticle surface, (b) number of Al nanoparticles in the irradiated area, and (c) electron beam flux. The carbon etched decreased with the increasing distance away (Figure 1A) from the nanoparticle concurring with the LSPR electric field decay, and the number of carbon atoms etched increased with the number of particles and electron beam flux (Figure 1B).

A spatial variation in carbon etching around the nanoparticles was also observed due to the local field enhancement by coupled LSP resonances (hot spots) between two or more nanoparticles clusters. Thickness maps of an area of. The thickness variation, indicated by vertical bars in Figure 1D), as measured using thickness maps of a three-nanoparticle cluster show that the highest carbon etching is confined to an area close to the interface where the nanoparticles are in contact (hot spots). These examples demonstrate that the knowledge gained from low-loss and core-loss EELS measurements can be used to design catalyst-plasmonic particle combinations for selected chemical reactions.

A

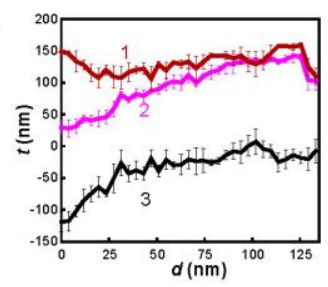

C

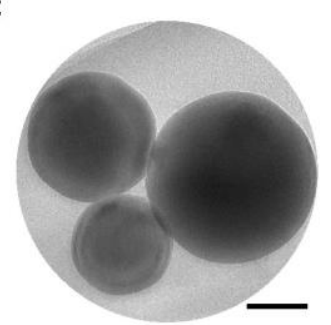

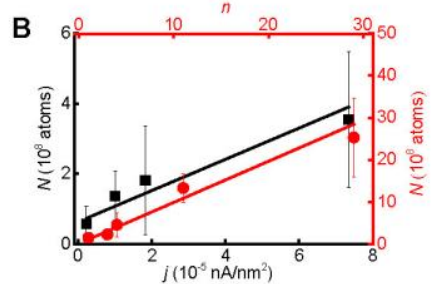

D

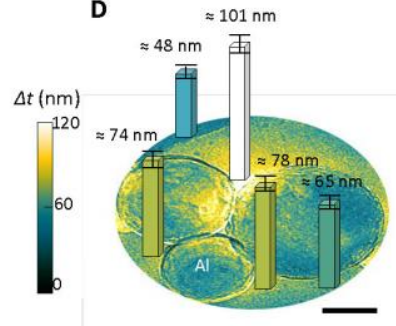

Figure 1. (A) Graphite film thickness change as a function of distance away from the surface of $\mathrm{Al}$ nanoparticle. (B) The number of carbon atoms etched as a function of electron flux (black) and the number of Al nanoparticles in the irradiated area (red). (C) TEM image showing a cluster of three Al particles. (D) Thickness difference map showing higher etching rate in the 'hot spot' regions (white and yellow vertical bars in the image) 


\section{References}

[1] M. Picher et al., Ultramicroscopy 150 (2015) 10.

[2] M. Picher et al., Nano Letters 14, (2014) 6104.

[3] W-C. D. Yang, Nature Materials 18 (2019), 614.

[4] S. Mukherjee, S. et al., J. American Chemical Society 136, (2014) 64.

[5] S. Linic, U. Aslam, C. Boerigter, and M. Morabito, Nature Materials 14, (2015) 567.

[6] Nicoletti, O. et al., Nature 502, 80-84 (2013).

[7] U Hohenester, Computer Physics Communications 185 (2014), p. 1177. 